\title{
Los deportes alternativos en el ámbito educativo
}

Sebastián Fierro Suero', Ainoa Haro Morillo² y Virginia García Montilla ${ }^{2}$

\section{'Graduado en Ciencias de la Actividad Física y del Deporte (Universidad de Huelva) \\ 2Alumna del Grado en Ciencias de la Actividad Física y del Deporte (Universidad de Huelva)}

\section{Email: chan994@gmail.com}

\section{RESUMEN:}

En este trabajo se pretende abordar el concepto de los deportes alternativos en el ámbito educativo. A lo largo de este artículo se va a realizar un acercamiento al concepto de deporte alternativo, tan utilizado y a la vez tan poco conocido. Vamos a introducirnos en él abordándolo desde diferentes puntos de vista, para tratar de conseguir una visión global sobre el pasado, presente y futuro de los deportes alternativos en las clases de educación física. Además, se analizará la relación entre el currículum y dicho concepto, para ver si su utilización en el marco educativo se corresponde con la consecución de los objetivos que se proponen. A su vez, se expondrá una propuesta de objetivos que deben cumplir los deportes alternativos para trabajarlos de forma eficaz. Por último, se concluye que el uso de los juegos y deportes alternativos en el ámbito educativo puede aportar muchos beneficios, ya que además de cumplir con los objetivos del currículum, suponen una novedad y resultan motivantes para todo el alumnado; aspecto que invita a seguir incluyéndolos en las clases de Educación Física.

PALABRAS CLAVE: Juegos Alternativos, Educación Física, Secundaria 


\section{INTRODUCCIÓN}

Los deportes alternativos son tema de debate en la actualidad. Defensores y detractores discuten sobre los beneficios y desventajas de la utilización de este tipo de actividades en el ámbito escolar. Sin embargo, como apuntan Robles, Abad, Castillo, Giménez y Robles (2013) parece ser que esta argumentación no es el factor que determina su empleo o no. Estos autores afirman que condicionantes como la formación recibida, las experiencias previas, o las instalaciones y los materiales disponibles son los factores que determinan la elección de uno u otro contenido en la educación física.

\subsection{Acercamiento conceptual y su aparición en España}

Pero... ¿Qué son los deportes alternativos? Antes de definir el concepto de deporte alternativo, vamos a tratar de aproximarnos al concepto en sí de deporte. Este concepto ha ido evolucionando a lo largo del tiempo, comenzando por definiciones más tradicionales y estrictas como la de Parlebas en 1981 (citado en Corrales, 2010) que lo definía como una situación motriz de competición institucionalizada.

Castejón (2001, citado por Robles, 2008, pp. 51-52) aporta una definición años después bastante más global en la que ya podríamos incluir los deportes alternativos:

actividad física donde la persona elabora y manifiesta un conjunto de movimientos o un control voluntario de los movimientos, aprovechando sus características individuales $\mathrm{y} / \mathrm{o}$ en cooperación con otro/s, de manera que pueda competir consigo mismo, con el medio o contra otro/s tratando de superar sus propios límites, asumiendo que existen unas normas que deben respetarse en todo momento y que también, en determinadas circunstancias, puede valerse de algún tipo de material para practicarlo.

Algunos autores en el último siglo han comenzado a definir el concepto de deporte alternativo. Barbero (2000) entiende como deporte alternativo todo aquel que se diferencia al tradicional, ya sea por el uso de un material diferente cuyo fin inicial no era la utilización actividad físico-deportiva, o bien pese a que ese si fuera su fin, se utiliza de forma diferente para la que fue diseñado originalmente. Por contra, un deporte tradicional/convencional siguiendo a Sauri (2005) sería practicado con las mismas herramientas y misma forma que se hacía en épocas pasadas, pudiendo adaptarse pero sin perder el modelo fijado que se ha trasmitido a lo largo del tiempo.

Hernández (2007) por su parte define el deporte alternativo como "aquel conjunto de deportes que, en contraposición con los modelos convencionales comúnmente aceptados, pretenden lograr un mayor carácter participativo y/o un explícito desarrollo de valores sociales, a través de modificaciones reglamentarias y/o la utilización de material novedoso". Este autor destaca además la importancia de la implicación del alumno en este tipo de deportes, ya que permite que se descubra así mismo mediante una participación activa en la que analiza, decide y ejecuta sus respuestas en un contexto lúdico.

Como afirma Requena (2008) los deportes alternativos suelen compartir características de los deportes cooperativos, pero además pueden presentar competición, sin llegar ésta a ser el objetivo, ya que se busca primar compartir frente a competir. 
Todas las definiciones de deportes alternativos siguen una misma idea, compartiendo un nexo en común y haciendo hincapié en aspectos como la participación, desarrollo de valores, mínima importancia al resultado competitivo, material novedoso, etc.

Según Dalmau y Peire (s.f.), el surgimiento de los deportes alternativos como corriente innovadora en España sucedió en la década de los 80. En esta eclosión tuvo una gran importancia la labor de la Federación Internacional del Deporte para todos, una institución internacional con sede en Bélgica y delegaciones en diferentes países del resto del mundo.

Por su parte, Jiménez (2010) propone que la aparición de los juegos alternativos en España surge a partir de los seminarios de INEF en Madrid en la década de los 90 . Originalmente estos seminarios se llamaron "Deportes alternativos para el año 2000" progresando luego al nombre de "Juegos y Deportes alternativos". Mediante estos seminarios se quería renovar los programas de Educación Física y acercar al concepto de "Deporte para Todos" gracias al uso de nuevos hábitos deportivos.

Siguiendo a Requena (2008), los juegos y deportes alternativos tienen un motivo de nacimiento común ya que aparecen por la gran institucionalización de los deportes tradicionales, que buscan el alto rendimiento. Por este motivo, se crean juegos y deportes que integren al alumnado en el deporte, sin importar sus capacidades físicas.

Ortí (2004) considera que muchos de estos deportes y juegos alternativos no son nuevos, sino que han venido siendo utilizados en otros ámbitos como en el recreativo y en el campo de la animación, casos de malabares o indiacas; u otros practicados en tiempo libre, como la petanca o las palas. Lo que es novedoso es la introducción de estos juegos y deportes alternativos en el currículo de la Educación Física. En este sentido, Ortí (2004) afirma que a pesar de esta tardía incorporación, este tipo de juegos y deportes presenta ciertas ventajas respecto a los contenidos tradicionales que se llevan a término.

Se podría decir en este aspecto, que los deportes alternativos aparecen en nuestro país en la década de los 90 proveniente de una corriente internacional relacionada con el concepto de "Deportes para Todos". Buscando esta integración global en el deporte, se traslada esta corriente al ámbito educativo, mediante la utilización de elementos inéditos en este campo hasta entonces.

Una vez clarificado el concepto y su aparición en España, vamos a conocer algunas propuestas de deportes alternativos que variarán notoriamente según cada autor. Por ejemplo, Ortí (2004) entiende el concepto de deporte alternativo desde una perspectiva más clásica, en la que incluye adaptaciones o modificaciones de deportes convencionales, como el fútbol o vóley playa, hockey alternativo, etc. También categoriza como deporte alternativo algunas actividades antes consideradas como juegos populares, como la petanca, la indiaca, el frisbee, las palas, malabares etc.

Por su parte Ruíz (1996) propone una batería más variada de juegos y deportes alternativos aplicables al ámbito educativo. En ella podemos encontrar algunos clásicos mencionados ya anteriormente, como frisbee, malabares, indiaca, paracaídas, etc. Sin embargo, este autor también propone la utilización de otros que consideramos más novedosos en las clases de Educación Física como balonkorf, ultimate, shuttleball, etc. A su vez, propone una clasificación separando en: 
- Juegos y deportes colectivos

- Floorball

- Lacrosse

- Fut-tenis

- Etc.

- Juegos y deportes de adversario

- Palas

- Peloc

- Etc.

- Deportes individuales de deslizamiento sobre rueda

- Bicicleta

- Monopatín

- Etc.

- Juegos de lanzamiento

- Boomerang

- Malabares

- Frisbee

- Etc.

- Juegos de cooperación

- Paracaídas

- Balones y globos gigantes

- Etc.

Encontramos que hay una tendencia de deportes alternativos nuevos que aplican diferentes autores como el caso del "flag football" (Requena, 2008); "kinball" (Lara y Cachón, 2010); "tchoukball” (Robles, 2009); "intercrosse" (Battaglia, 2013), etc.

Observamos que hay una propensión continua por parte de algunos autores en el desarrollo de juegos y deportes alternativos para tratar de solventar, o incluso, complementar las necesidades de la Ley Educativa. En este sentido, esa es la razón de aparición de juegos como el "intercrosse", que trata de disminuir el contacto físico de su predecesor "lacrosse".

\subsection{Conexión curricular}

Teniendo una idea ya más sólida de lo que son los deportes alternativos, vamos a tratar de comprender qué papel juegan estos en el ámbito educativo y en la propia legislación. Siguiendo a Ruiz (1996), la sociedad demanda al sistema educativo una calidad de la enseñanza que hace actualizar la metodología, los recursos didácticos, la formación continua y los nuevos contenidos, proponiendo nuevas alternativas en el área correspondiente. Una de las formas para contribuir a esto es a través de los juegos y deportes alternativos, que cada vez son más habituales en el área de Educación Física.

Siguiendo con la idea de Ruiz (1996), una de las razones de peso por las que trabajar a través de estos juegos y deportes alternativos es que ofrecen nuevas posibilidades educativas, recreativas, de ejercicio y diversión, evitando la rutina y la poca motivación que puede producir la práctica única y reiterada de deportes convencionales. Otra de las causas por las cuales introducir éstos en las clases de educación física es por su material asequible, tanto económicamente como fáciles de adquirir. Lara y Cachón (2010) afirman que los profesores de educación física pueden emplear este tipo de deporte de forma muy diversa con objetivo de "obtener resultados diversos, ya sean de desarrollo motriz, intelectual, cooperación, coeducación, colaboración, compromiso, formación de valores..." 
A continuación tratamos de justificar la utilización del deporte y en especial del deporte alternativo en la Educación Secundaria, para ello vamos a tratar de relacionar los objetivos propuestos, primeramente con la Ley Orgánica 2/2006, de 3 de mayo, y en el Real Decreto 1631/2006, de 29 de diciembre a nivel nacional en el que podemos leer:

“Artículo 3: Objetivos de la Educación secundaria obligatoria en España.

- Asumir (...), practicar la tolerancia, la cooperación y la solidaridad entre las personas y grupos, ejercitarse en el diálogo afianzando los derechos humanos como valores comunes de una sociedad plural (...)."

De la misma forma, lo relacionamos ahora con el Decreto 231/2007, del 31 de Julio en el que se establece la ordenación y las enseñanzas correspondientes a la Educación Secundaria Obligatoria en Andalucía. En este documento se puede leer:

"Artículo 4: Objetivos de la Educación secundaria obligatoria en Andalucía.

a. Adquirir habilidades que les permitan desenvolverse con autonomía (...) así como en los grupos sociales con los que se relacionan, participando con actitudes solidarias, tolerantes y libres de prejuicios.

b. Interpretar y producir con propiedad, autonomía y creatividad mensajes que utilicen códigos artísticos, científicos y técnicos."

“Artículo 5. Definición y principios para su determinación.

4. Asimismo, el currículo incluirá:

a. El fortalecimiento del respeto de los derechos humanos y de las libertades fundamentales y los valores que preparan al alumnado para asumir una vida responsable en una sociedad libre (...).

c. Contenidos y actividades para la adquisición de hábitos de vida saludable y deportiva y la capacitación para decidir entre las opciones que favorezcan un adecuado bienestar físico, mental y social, para el propio alumno o alumna y para los demás."

Pese a que podemos justificar la utilización de los deportes alternativos en el ámbito educativo mediante el currículum, tanto a nivel nacional como autonómico, esta justificación no va más allá de la que podríamos hacer en relación a los deportes convencionales. Es decir, bajo nuestro criterio, las leyes educativas legítimas no guardaban suficiente concordancia con la realidad educativa. Estas leyes, deberían ser más cercanas, mirar más por el desarrollo integral del alumnado.

Si nos centramos en la normativa estatal y autonómica vigente, en el RD 1105/2014, de 26 de diciembre, por el que se establece el currículo básico de la Educación Secundaria Obligatoria y del Bachillerato, se puede leer el siguiente objetivo:

"K) Conocer y aceptar el funcionamiento del propio cuerpo y el de los otros, respetar las diferencias, afianzar los hábitos de cuidado y salud corporales e incorporar la educación física y la práctica del deporte para favorecer el desarrollo personal y social."

Este objetivo también aparece en el Decreto 111/2016 donde se establece el currículo para la ESO en Andalucía y en él se hace mención explícita a la Educación 
Física y la práctica de deporte como medio de desarrollo personal y social. En esta misma línea, en el Decreto 110/2016 (currículo de Bachillerato de Andalucía) aparece el objetivo "m) Utilizar la educación física y el deporte para favorecer el desarrollo personal y social". Además, llama la atención de forma muy positiva que en la Orden de 14 de julio de 2016 (donde se desarrolla el currículo correspondiente a la ESO), aparezcan como contenidos a trabajar los juegos y deportes alternativos. En el bloque de juegos y deportes se incluyen contenidos como los juegos predeportivos, los deportes, etc. Así, entre los contenidos a tratar en $1^{\circ}$ de ESO se incluye "Juegos alternativos, como por ejemplo: hockey escolar, ultimate, rugby escolar, etc". En $2^{\circ}$ de ESO, entre los contenidos a trabajar en el bloque 3 (Juegos y Deportes) en Andalucía aparece: "Juegos alternativos y predeportivos". Por su parte en $3^{\circ} \mathrm{ESO}$, se incluye el mismo contenido "Juegos aternativos y predeportivos" y en $4^{\circ}$ de ESO aparece de nuevo el contenido "Juegos alternativos". Por lo que en Andalucía, se ha recogido expresamente como un contenido más a trabajar durante la ESO, lo que parece consolidar la importancia y utilidad de este tipo de juegos y deportes para la formación integral de los jóvenes estudiantes.

Basándonos en diversos autores como Arráez (1995), Jiménez (2010), Fernández (2010) y Robles (2009), así como nuestra propia aportación tras el análisis anterior, hemos creado los siguientes objetivos que consideramos acordes para los juegos y deportes alternativos en la clase de educación física y de los que podrían sacarse aspectos para añadir a los objetivos de futuras leyes educativas. Estos son:

- Presentar situaciones motrices nuevas, motivantes y lúdicas que ayuden a contribuir a la consecución de una educación óptima en el ámbito escolar, prevaleciendo el aspecto recreativo sobre el competitivo.

- Utilizar los juegos y deportes alternativos como instrumento para el fomento de la igualdad de oportunidades y aumento de la motivación de los grupos de alumnos menos participativos.

- Fomentar mediante las nuevas actividades motrices la aparición de valores en el aula, tales como: el compañerismo, el respeto, la cooperación, la igualdad, etc.

- Introducir materiales no específicos en el aula con nuevas posibilidades de uso, así como actividades que no requieren de instalaciones convencionales para su desarrollo.

- Emplear los juegos alternativos como método de iniciación a deportes convencionales que requieren mayor complejidad.

- Mejorar las habilidades motrices mediante la utilización de estas nuevas actividades.

Es indispensable que la aplicación de los deportes alternativos, como cualquier otro elemento novedoso a nivel educativo, siga una correcta programación y cumpla con unos objetivos para reportar las mejoras esperadas.

\section{ESTADO ACTUAL Y CONCLUSIONES}

En la última década Parra y Sancho (2008) apuntan que los llamados deportes tradicionales han pasado a ser poco motivantes para el alumnado en las clases de Educación Física, sobre todo, debido al exceso de utilización de ellos por parte del profesorado. La búsqueda de nuevas experiencias y la propia desmotivación de los 
alumnos han hecho que surjan nuevos juegos y deportes que satisfagan esas inquietudes.

Según Lara y Cachón (2010), los deportes alternativos han sido muy usados en los últimos años; sin embargo, hay un sector dentro del profesorado que, ya sea por desconocimiento o por falta de documentación, no los usa asiduamente. Por tanto, estos autores apuntan que los beneficios de estos juegos y deportes en la clase de educación física no han llegado a los niveles esperados, debido a su todavía baja utilización; aunque es cierto que la utilización de este recurso es claramente ascendente. En la misma línea Hernández (2007) afirma "en las últimas décadas, parte del profesorado de Educación Física ha ido incorporando actividades alternativas que han sido capaces de romper la tendencia a imperar de una única orientación deportiva, favoreciendo nuevas perspectivas de desarrollo".

Podríamos decir que desde sus comienzos en las décadas de los 80 y 90, los juegos alternativos han ido incrementando su uso considerablemente en todos los ámbitos. Concretamente, en el ámbito escolar vemos cómo ha ido progresando originalmente con una perspectiva más recreativa, donde se buscaba situaciones lúdicas y novedosas con materiales hasta entonces desconocidos en este ámbito, como por ejemplo las palas, los malabares, la indiaca, etc. Para evolucionar hacia una perspectiva más centrada en la educación, sin dejar de lado el aspecto lúdico, pero basándose en la consecución de unos objetivos didácticos establecidos. En este sentido, se ha realizado una propuesta de objetivos de los deportes alternativos para facilitar su inclusión en el ámbito educativo. Nuestra propuesta ha ido en la línea de diversos autores que se centran en aspectos como la igualdad de oportunidades, compañerismo, valores educativos, etc. Buscan una nueva perspectiva que cubra el déficit que no eran capaces de solventar los juegos o deportes hasta ahora trabajados, en los que la competición ocupaba una gran base, lo que en muchas ocasiones hacía que sólo participaran y resultaran motivantes para aquellos alumnos con mayor capacidad físico-motriz.

A su vez, los objetivos perseguidos por estos juegos están en concordancia con el currículum tanto a nivel nacional, como autonómico, tal y como se refleja en los fragmentos del currículum antes citados en el apartado correspondiente. Además, la nueva normativa andaluza (Orden de 14 de julio de 2016) aboga expresamente por el uso de juegos y deportes alternativos durante las clases de EF (correspondientes a la Educación Secundaria Obligatoria).

Por estas razones, defendemos el uso de los juegos y deportes alternativos en el ámbito educativo, ya que además de cumplir con los objetivos del currículum, llevados a cabo de forma correcta, resultan motivantes para todos los alumnos. Por otra parte, la propia experiencia de los profesores que los están llevando a cabo es satisfactoria, aspecto que invita a seguir incluyéndolos en las clases de Educación Física.

Pese a la rotunda defensa de la introducción de los juegos y deportes alternativos en el ámbito escolar, no queremos dejar de lado a los juegos y deportes convencionales, ya que estos son de vital importancia para cumplir las necesidades 
curriculares. El conjunto de ambos, supone la complementación necesaria para desarrollar los contenidos pedagógicos. Por tanto, como dice Hernández (2007) "La herramienta está creada, ahora está en nuestras manos el saber utilizarla".

\section{REFERENCIAS}

Arráez, J. M. (1995). Juegos y deportes alternativos con deficientes psíquicos. Apunts: Educación física y deportes, 40, 69-82.

Barbero, J. C. (2000). Los juegos y deportes alternativos en Educación Física. Lecturas: Educación Física y Deportes, Revista Digital, 22. Recuperado de http://www.efdeportes.com/efd22a/altern.htm

Battaglia, L. C. (2013). El intercrosse, una propuesta escolar. En el $10^{\circ}$ Congreso Argentino y 5o Latinoamericano de Educación Física y Ciencias, 9 al 13 de septiembre de 2013, La Plata (Argentina). Recuperado de http://www.memoria.fahce.unlp.edu.ar/trab eventos/ev.3105/ev.3105.pdf

Castejón, F. J. (1994). La enseñanza del deporte en la educación obligatoria: enfoque metodológico. Revista Complutense de Educación, 5(2), 137-152.

Corrales, A. R. (2010). El deporte como elemento educativo indispensable en el área de educación física. EmásF, Revista Digital de Educación Física, 4, 23-36. Recuperado de http://emasf2.webcindario.com/NUMERO 4.pdf

Dalmau, J. M. y Peire, T. (s.f.). Curso CPR: De los juegos alternativos a los nuevos deportes. Recuperado: 12/05/2016 de http://multiblog.educacion.navarra.es/imoreno1/files/2010/06/Apuntes-de-textodel-curso.pdf

Decreto 231/2007, del 31 de Julio en el que se establece la ordenación y las enseñanzas correspondientes a la Educación Secundaria Obligatoria en Andalucía. Boletín Oficial de la Junta de Andalucía. Sevilla, 8 de agosto de 2007, núm. 156, pp. 15-25.

Decreto 110/2016, de 14 de junio, por el que se establece la ordenación y el currículo del Bachillerato en la Comunidad Autónoma de Andalucía. Boletín Oficial de la Junta de Andalucía. Sevilla, 28 de junio de 2016, núm. 122, pp. 11-27.

Decreto 111/2016, de 14 de junio, por el que se establece la ordenación y el currículo de la Educación Secundaria Obligatoria en la Comunidad Autónoma de Andalucía. Boletín Oficial de la Junta de Andalucía. Sevilla, 28 de junio de 2016, núm. 122, pp. 27-46.

Fernández, R. F. (2010). Juegos innovadores con material alternativo en el área de Educación Física. EmásF: revista digital de educación física, 4, 5-18. Recuperado de http://emasf2.webcindario.com/NUMERO 4.pdf

Hernández, J. M. (2007). El valor pedagógico de los deportes alternativos. Lecturas: Educación Física y Deportes, Revista Digital, 114. Recuperado de http://www.efdeportes.com/efd114/el-valor-pedagogico-de-los-deportesalternativos.htm

Jiménez, J. (2010). Los juegos y deportes alternativos en educación física. Revista Digital Enfoques Educativos, 67, 142-151.

Lara, A. J., y Cachón, J. (2010). Kinball: los deportes alternativos en la formación del/la docente de educación física. En CiDd: I/ Congrés Internacional de Ditáctiques 
2010, Girona (Universitat de Girona). Recuperado de http://dugidoc.udg.edu/bitstream/handle/10256/3001/491.pdf?sequence $=1$

Ley Orgánica 2/2006, de 3 de mayo, de Educación. Boletín Oficial del Estado. Madrid, 4 de mayo de 2006, núm. 2103, pp. 97858-97921.

Orden 14 de julio de 2016, por la que se desarrolla el currículo correspondiente a la Educación Secundaria Obligatoria en la Comunidad Autónoma de Andalucía, se regulan determinados aspectos de la atención a la diversidad y se establece la ordenación de la evaluación del proceso de aprendizaje del alumnado. Boletín Oficial de la Junta de Andalucía. Sevilla, 28 de julio de 2016, núm. 144, pp. 108-396.

Ortí, J. (2004). La animación deportiva, el juego y los deportes alternativos. Barcelona: Inde.

Parra, C. y Sancho, M. (2008). Deportes alternativos: una propuesta práctica desde el punto de vista del profesor. En $V$ congreso nacional y III congreso iberoamericano de deporte en edad escolar: "Nuevas Tendencias $y$ perspectivas de futuro" (pp. 351-374), 20, 21 y 22 de noviembre de 2008, Dos Hermanas (Sevilla). Recuperado de http://www.munideporte.com/imagenes/ documentacion/ficheros/20081224125537deporte escolar.pdf

Real Decreto 1631/2006, de 29 de diciembre, por el que se establecen las enseñanzas mínimas correspondientes a la Educación Secundaria Obligatoria. Boletín Oficial del Estado. Madrid,5 de enero de 2007, núm. 5, pp. 677-773.

Real Decreto 1105/2014, de 26 de diciembre, por el que se establece el currículo básico de Educación Secundaria Obligatoria y del Bachillerato. Boletín Oficial del Estado. Madrid, 3 de enero de 2015, núm. 3, pp. 169-546.

Requena, Ó. (2008). Juegos alternativos en educación física: flag football. Revista Digital Innovación y Experiencias Educativas, 4. Recuperado de http://docplayer.es/20871787-Juegos-alternativos-en-educacion-fisica-flagfootball.html

Robles, J. (2008). Tratamiento del deporte dentro del área de educación física durante la etapa de Educación Secundaria Obligatoria en la provincia de Huelva (Tesis doctoral). Huelva: Universidad de Huelva. Recuperado de http://rabida.uhu.es/dspace/bitstream/handle/10272/153/b15153630.pdf?seque $\underline{\text { nce }=1}$

Robles, J. (2009). Tchoukball. Un deporte de equipo novedoso: propuesta de aplicación en Secundaria. Retos, 16, 75-79.

Robles, J., Abad M. T., Castillo, E., Giménez, F. J., Robles, A. (2013). Factores que condicionan la presencia de la expresión corporal en la enseñanza secundaria según el profesorado de educación física. Retos, 24, 171-175.

Ruíz, J. G. (1996). Juegos y deportes alternativos en la programación de Educación física escolar. Editorial Deportiva Agonos. Lérida.

Sauri, C. (2005): Juegos tradicionales, del currículum a la clase: teoría y práctica para la aplicación en Educación Física. Sevilla: Wanceulen. 